\title{
Observational constraints on cosmic string production during brane inflation
}

\author{
Levon Pogosian, ${ }^{1}$ S.-H. Henry Tye, ${ }^{2}$ Ira Wasserman, ${ }^{2,3}$ and Mark Wyman ${ }^{2,3}$ \\ ${ }^{1}$ Theoretical Physics, The Blackett Laboratory, Imperial College, London SW7 2BZ, United Kingdom \\ ${ }^{2}$ Laboratory for Elementary Particle Physics, Cornell University, Ithaca, New York 14853, USA \\ ${ }^{3}$ Center for Radiophysics and Space Research, Cornell University, Ithaca, New York 14853, USA
}

(Received 26 April 2003; published 3 July 2003)

\begin{abstract}
Overall, brane inflation is compatible with the recent analysis of the Wilkinson microwave anisotropy probe (WMAP) data. Here we explore the constraints of WMAP and the 2dF Galaxy Redshift Survey (2dFGRS) data on the various brane inflationary scenarios. Brane inflation naturally ends with the production of cosmic strings, which may provide a way to distinguish these models observationally. We argue that currently available data cannot exclude a non-negligible contribution from cosmic strings definitively. We perform a partial statistical analysis of mixed models that include a subdominant contribution from cosmic strings. Although the data favor models without cosmic strings, we conclude that they cannot definitively rule out a cosmic-stringinduced contribution of $\sim 10 \%$ to the observed temperature, polarization and galaxy density fluctuations. These results imply that $G \mu \lesssim 1.3 \times 10^{-6} \sqrt{B \lambda / 0.1}$, where $\lambda \leqslant 1$ is a measure of the intercommutation probability of the cosmic string networks and $B$ measures the importance of perturbations induced by cosmic strings. We argue that, conservatively, the data available currently still permit $B \leqq 0.1$. Precision measurements sensitive to the $B$-mode polarization produced by vector density perturbation modes driven by the string network could provide evidence for these models. Accurate determinations of $n_{s}(k)$, the scalar fluctuation index, could also distinguish among various brane inflation models.
\end{abstract}

DOI: 10.1103/PhysRevD.68.023506

PACS number(s): $98.80 . \mathrm{Cq}$

\section{INTRODUCTION}

Observations of the cosmic microwave background (CMB) $[1,2]$ support the idea that the standard big bang phase of the expansion of the universe was preceded by inflation [3]. Recent results from the Wilkinson microwave anisotropy probe (WMAP) [4-8] constrain the properties of proposed inflationary models tightly, but although some models are now excluded, numerous possibilities remain. A further challenge to observational cosmology is to try to hone in on a small class of viable models, even if identifying a single, correct theory of inflation may prove impracticable.

All of the data collected up until now are consistent with a relatively pristine universe in which the perturbations observed today result from the amplification and distortion of a relatively featureless, Gaussian spectrum of fluctuations produced by quantum effects during inflation. However, it is likely that inflation itself could have left behind other remnants-such as cosmic strings-which could actively perturb both the CMB and dark matter of the universe up to the present day.

It is well known that cosmic strings cannot be wholly responsible for either the CMB temperature fluctuations or the observed clustering of galaxies [9]; roughly speaking, the implied limits on the cosmic string tension $\mu$ allowed by observations is $G \mu \lesssim 10^{-6}$. However, now that cosmology has entered an era in which the properties of the universe are being revealed to unprecedented precision, a natural question is to what extent the observations can allow previously unwanted ingredients, such as cosmic strings (e.g., [10]). Indeed, as the precision of cosmological observations increases, we might hope to be able to distinguish among numerous presently viable models for inflation by the properties of the cosmic strings they predict.
Although the idea that inflationary cosmology might lead to cosmic string formation is not new [11], it has received new impetus from the brane world scenario suggested by superstring theory. In brane world cosmology, standard model particles and interactions correspond to open string (brane) modes, while the graviton, the dilaton and the radions are closed string (bulk) modes. Thus, our 3D universe can be thought of as residing on a brane or stack of branes with three dimensions of cosmological size. These branes in turn reside in extra dimensions that are compactified. In such a model, inflation can result during the collisions of branes coalescing to form, ultimately, the brane on which we live [12].

In these brane inflation models, the separations between branes in the compactified dimensions are scalar fields (open string modes) that can act as inflatons, with the interaction potential between spatially separated branes providing the inflaton potential. Details of the brane inflation scenario depend on both qualitative and quantitative features, such as whether collisions involve a brane-antibrane pair [13] or two branes coalescing at an angle [14], as well as parameters such as the sizes of the compactified dimensions $[15,16]$. Qualitatively, though, it appears easy to find models that predict adiabatic temperature and dark matter fluctuations capable of reproducing all currently available observations. A seemingly unavoidable outcome of brane inflation, though, is the production of a network of cosmic strings [16,17], whose effects on cosmological observables ranges from negligible to substantial, depending on the specific brane inflationary scenario [18].

Although cosmic string production towards the end of inflation is possible in field theory models [11], the scaling properties of the cosmic string networks in brane inflationary scenarios are different than that in the familiar $(3+1) \mathrm{D}$ 
simulations, since intercommutation probabilities are smaller as a consequence of the existence of extra dimensions. In addition to placing constraints on the amplitude of string induced perturbations of the $\mathrm{CMB}$, we show that the results place limits on $G \mu / \sqrt{\lambda}$, where $\mu$ is the string tension and $\lambda \leqslant 1$ is a dimensionless measure of the intercommutation rate.

Here we shall first review the essential points of brane inflation, and examine the constraints imposed by the WMAP observations if we ignore the contribution from cosmic strings. These constraints allow us to delineate a range of possible cosmic string tensions. Then, we assess quantitatively the extent to which cosmic strings can contribute to the CMB temperature fluctuations and power spectra of dark matter density perturbations. In this analysis, we hold properties of the background cosmological model fixed to their best fit values, as determined by WMAP [5] without cosmic strings. (A more detailed analysis that varies the background cosmology as well is under way.) Although the available data favor models without cosmic strings, they may still allow, within the uncertainties, a contribution from string-induced perturbations of up to $10 \%$. They also imply scalar perturbation indices $n_{s}(k)$ which, although still consistent with a broad range of models, may be able to discriminate among them in future. We also compute the dark matter density perturbation power spectrum, and compare with observational determinations from the 2dFGRS galaxy survey [19]. We discuss the interpretation of these results in terms of the string tension and efficiency with which the cosmic string network decays via intercommutation of string segments, which is reduced in a universe with extra dimensions. Finally, we discuss the prospects for detecting $B$-mode polarization, which is expected to be a prominent signature of a cosmic string network, in view of the constraints implied by our analysis.

\section{BRANE INFLATION AND COSMIC STRING PROPERTIES}

Recently, the brane world scenario suggested by superstring theory was proposed, where the standard model of the strong and electroweak interactions are open string (brane) modes while the graviton and the radions are closed string (bulk) modes. The relative brane positions (i.e., brane separation) in the compactified dimensions are scalar fields that have just the right properties to act as inflatons. Thus, the brane inflation scenario emerges naturally in the brane world [12]. In this picture, the inflaton potential is due to the exchange of closed string modes between branes; this is the dual of the one-loop partition function of the open string spectrum, a property well studied in string theory [20]. This interaction is of gravitational strength, resulting in a very weak (flat) potential, ideally tailored for inflation.

The potential is essentially dictated by the attractive gravitational (and the Ramond-Ramond) interaction between branes. As the branes move towards each other, slow-roll exponential inflation takes place. This yields an almost scaleinvariant power spectrum for density perturbation, except there is a slight red tilt (at a few percent level). As they reach a distance around the string scale, the inflaton potential becomes quite steep so that the slow-roll condition breaks down. At around the same time, a complex tachyon appears, so inflation ends rapidly as the tachyon rolls down its potential. In effect, inflation ends when the branes collide, heating the universe to start the standard big bang phase of cosmological expansion. This brane inflationary scenario may be realized in a number of ways $[15,16]$. The scenario is simplest when the radion and the dilaton (bulk) modes are assumed to be stabilized by some unknown non-perturbative bulk dynamics at the onset of inflation. Since the inflaton is a brane mode, and the inflaton potential is dictated by the brane mode spectrum, it is reasonable to assume that the inflaton potential is insensitive to the details of the bulk dynamics.

Coupling of the tachyon to inflaton and standard model fields can allow efficient heating of the universe if certain conditions on the coupling of the tachyon to standard model particles are met [21]. As the tachyon rolls down its potential, besides heating the universe, the vacuum energy also goes to the production of defects, in particular, cosmic strings. The effect of the resulting cosmic string network may be negligible or rather substantial, depending on the particular brane inflationary scenario [18]. However, in all cases, we expect the density perturbation power spectrum in the CMB to be dominated by the adiabatic fluctuations arising from quantum fluctuations of the inflaton during brane inflation, not by the nonadiabatic contributions from cosmic strings. However, the contribution to the density perturbation power spectrum in the CMB coming from the cosmic string network may be large enough to be observable.

We devote this section to a review of the implications of brane inflation. For a broad set of models, we present results for the slow roll evolution, fluctuation spectra, string mass scale, and associated cosmic string tension. (These results follow directly from the treatments in Refs. [13,15-18].) We consider the collision of a $\mathrm{D} p$ brane with a $\mathrm{D} p$ brane at an angle $\theta$; collision with a $\mathrm{D} p$ antibrane corresponds to $\theta$ $=\pi$. (Here and throughout this section, we follow [16], which contains more details and discussion.) Of the ten spacetime dimensions, one is the time, three are the large spatial dimensions we live in, and the rest are compactified. Of the compact dimensions, $p-3$ are parallel to the brane, and we take their compactification lengths to be $\ell_{\|}=2 \pi r_{\|}$, implying a volume $V_{\|}=\ell^{p-3}$. Of the remaining $d=9-p$ dimensions, we take $d-d_{\perp}$ to be compactified with a size $2 \pi / M_{s}$, where $M_{s}$ is the string scale, while the remaining $d_{\perp}$ are compactified with a size $\ell_{\perp}=2 \pi r_{\perp}>2 \pi / M_{s}$. The 10 -dimensional gravitational coupling constant is

$$
\kappa^{2}=8 \pi G_{10}=\frac{g_{s}^{2}(2 \pi)^{7}}{2 M_{s}^{8}},
$$

where $g_{s}$ is the expectation value of the dilatonic string coupling, which is related to the standard model gauge coupling $\alpha\left(r_{\|}\right)$on a scale $1 / r_{\|}$by

$$
g_{s}=2\left(M_{s} r_{\|}\right)^{p-3} \alpha\left(r_{\|}\right)
$$

the 4-dimensional Planck scale $M_{P}=(8 \pi G)^{-1 / 2}$ is then 


$$
g_{s}^{2} M_{P}^{2}=\frac{M_{s}^{2}\left(M_{s} r_{\perp}\right)^{d_{\perp}}\left(M_{s} r_{\|}\right)^{p-3}}{\pi} .
$$

The outcome of brane inflation will therefore depend on several parameters, $p, d_{\perp}, r_{\perp}, r_{\|}$and $\alpha\left(r_{\|}\right)$.

We will distinguish between two different potentials for the interaction between branes, depending on their separations. (See [13-15].) For some scenarios, a fixed lattice of branes is considered to be spread throughout the compactified dimensions, with a moving brane placed inside one lattice square. At separations small compared to the lattice size of the compactification topology, the interaction is "Coulombic," with a potential of the form $V(y)=V_{0}-U / y^{d_{\perp}-2}$ for a separation $y$ in the large compact dimensions. This potential is suitable for inflation resulting from the collision of a pair of relatively nearby branes at a small angle [14]. When the separation is nearly equal to the lattice size, an expansion about zero displacement from the anti-podal point gives $V(y)=V_{0}-U^{\prime} y^{\sigma}$, where $\sigma$ depends on the compactification topology. This potential is suitable for the brane-antibrane scenario (which corresponds to branes at an angle $\pi$ ). In the next two sections, we summarize the inflation scenario for interbrane potentials of these two general forms.

\section{A. Coulombic inflation}

Consider a potential of the form

$$
V(\psi)=V_{0}\left(1-\frac{\eta}{\left(d_{\perp}-2\right) \psi^{d_{\perp}-2}}\right),
$$

with $\psi \propto y$, the interbrane spacing; for the special case $d_{\perp}$ $=2$ this becomes a logarithmic potential, but the results we derive below may be applied to this special case. (We only consider $d_{\perp}-2 \geqslant 0$ here to simplify our analysis, since the results generalize easily to the logarithmic case.) In the slow roll approximation, the equation of motion for $\psi$ becomes

$$
\frac{d \psi}{d L}=-\frac{\eta M_{P}^{2}}{\psi^{d_{\perp}-1}}
$$

where $L=\ln a$ is the logarithm of the scale factor $a(t)$, which we consider to be zero at the start of inflation. The slow roll solution is then

$$
\begin{aligned}
\psi & =\left[\psi_{i}^{d_{\perp}}-d_{\perp} \eta M_{P}^{2} L\right]^{1 / d_{\perp}}=\left[d_{\perp} \eta M_{P}^{2}\left(L_{\mathrm{inf}}-L\right)\right]^{1 / d_{\perp}} \\
& \equiv\left(d_{\perp} \eta M_{P}^{2} L_{r}\right)^{1 / d_{\perp}}
\end{aligned}
$$

where the starting value of the field is $\psi_{i}$, the total number of $e$-folds in inflation is

$$
L_{\mathrm{inf}}=\frac{\psi_{i}^{d_{\perp}}}{d_{\perp} \eta M_{P}^{2}}
$$

and $L_{r}=L_{\text {inf }}-L$ is the total number of $e$-folds remaining in inflation. The curvature fluctuation spectrum is then

$$
\Delta_{\mathcal{R}}^{2}(k)=\frac{H^{4}}{4 \pi^{2} \dot{\psi}^{2}}=\frac{V_{0}\left(d_{\perp} L_{r}\right)^{2\left(1-1 / d_{\perp}\right)}}{12 \pi^{2} \eta^{2 / d_{\perp}} M_{P}^{2+4 / d_{\perp}}},
$$

where $L_{r}$ is evaluated when $k / a=H$ or $\ln \left(k / k_{0}\right)=L_{r, 0}-L_{r}$, where $k_{0}$ is a reference scale, which crosses with $L_{r, 0} e$-folds remaining in inflation. The fluctuation spectrum is very flat, with only slowly varying scalar index $n_{s}(k)$,

$$
\begin{aligned}
n_{s}(k)-1 & =\frac{d \ln \Delta_{\mathcal{R}}^{2}(k)}{d \ln k}=-\frac{2}{L_{r}(k)}\left(1-\frac{1}{d_{\perp}}\right) \\
& \simeq-0.03\left[\frac{60}{L_{r}(k)}\right]\left(1-\frac{1}{d_{\perp}}\right) \\
\frac{d n_{s}(k)}{d \ln k} & =-\frac{2}{L_{r}^{2}(k)}\left(1-\frac{1}{d_{\perp}}\right) \\
& \simeq-6 \times 10^{-4}\left[\frac{60}{L_{r}(k)}\right]^{2}\left(1-\frac{1}{d_{\perp}}\right),
\end{aligned}
$$

both of which are in the range of uncertainty of the determinations in [5].

The challenge to this, or any other, inflation model is to have sufficient inflation as well as small curvature fluctuation. Since the precise value of $L_{\text {inf }}$ depends on initial conditions as well as on parameters of the model, let us first consider the constraints on the latter implied by comparing Eq. (8) to the WMAP result $\Delta_{\mathcal{R}}^{2}\left(k_{0}\right)=2.95 \times 10^{-9} A\left(k_{0}\right)$ with $A\left(k_{0}\right)=0.9 \pm 0.1$. To do this, let us consider a particular model with $p=4$ and a small collision angle $\theta$; then we have

$$
\psi=y \sqrt{\frac{\tau_{4} \ell_{\|}}{2}}
$$

$$
V_{0}=\frac{\tau_{4} \ell_{\|} \theta^{2}}{4}
$$

$$
\begin{aligned}
\frac{\tau_{4} \ell_{\|}}{2} & =\frac{M_{s}^{4}}{32 \pi^{3} \alpha\left(r_{\|}\right)} \\
\eta & =\frac{\beta\left(d_{\perp}\right)}{16 \pi} \theta M_{s}^{6-d_{\perp}}\left(\frac{\tau_{4} \ell_{\|}}{2}\right)^{\left(d_{\perp}-4\right) / 2}
\end{aligned}
$$

$$
\Delta_{\mathcal{R}}^{2}(k)=\frac{\left(\theta d_{\perp} L_{r}\right)^{2\left(1-1 / d_{\perp}\right)}}{24\left[64 \beta\left(d_{\perp}\right)\right]^{2 / d_{\perp}} \pi^{2+10 / d_{\perp}}\left[\alpha\left(r_{\|}\right)\right]^{4 / d_{\perp}}}\left(\frac{M_{s}}{M_{P}}\right)^{2+4 / d_{\perp}},
$$

where 


$$
\begin{gathered}
\tau_{p}=\frac{M_{s}^{p+1}}{(2 \pi)^{p} g_{s}} \\
\beta=\left\{\begin{array}{ccc}
\frac{1}{2 \pi^{d_{\perp} / 2}}\left(\frac{\Gamma d_{\perp}-2}{2}\right) & d_{\perp}>2 \\
\frac{1}{\pi} & d_{\perp}=2 .
\end{array}\right.
\end{gathered}
$$

Let us consider the specific example $d_{\perp}=2$; for this case we find

$$
\Delta_{\mathcal{R}}^{2}(k)=\frac{\theta L_{r}}{768 \pi^{6}\left[\alpha\left(r_{\|}\right)\right]^{2}}\left(\frac{M_{s}}{M_{P}}\right)^{4} \quad\left[d_{\perp}=2\right],
$$

and therefore the string scale is determined to be

$\frac{M_{s}}{M_{P}} \simeq 2.5 \times 10^{-2}\left[25 \alpha\left(r_{\|}\right)\right]^{1 / 2}\left[A\left(k_{0}\right)\right]^{1 / 4}\left(\frac{10}{\theta L_{r}}\right)^{1 / 4} \quad\left[d_{\perp}=2\right]$,

that is, of the same order of energy as the grand unified theory (GUT) scale, $10^{15} \mathrm{GeV}$. Larger $d_{\perp}$ leads to smaller $M_{s} / M_{P}$; thus if $d_{\perp}=4$ we find

$$
\Delta_{\mathcal{R}}^{2}(k)=\frac{\left(\theta L_{r}\right)^{3 / 2}}{12 \sqrt{2} \pi^{7 / 2} \alpha\left(r_{\|}\right)}\left(\frac{M_{s}}{M_{P}}\right)^{3} \quad\left[d_{\perp}=4\right]
$$

which in turn requires

$$
\frac{M_{s}}{M_{P}} \simeq 1.6 \times 10^{-3}\left[25 \alpha\left(r_{\|}\right)\right]^{1 / 3}\left[A\left(k_{0}\right)\right]^{1 / 3}\left(\frac{10}{\theta L_{r}}\right)^{1 / 2} \quad\left[d_{\perp}=4\right] .
$$

The total number of $e$-folds in inflation is

$$
\begin{aligned}
L_{\mathrm{inf}}= & \frac{\left(M_{s} y_{i}\right)^{d_{\perp}} M_{s}^{2}}{64 \pi^{5} \beta\left(d_{\perp}\right) \theta\left[\alpha\left(r_{\|}\right)\right]^{2} M_{P}^{2}} \\
= & \frac{\pi^{\left[d_{\perp}\left(d_{\perp}-1\right)\right] /\left(d_{\perp}+2\right)}\left(2 \zeta_{i} M_{s} r_{\perp}\right)^{d_{\perp}}}{\theta^{3 d_{\perp} /\left(d_{\perp}+2\right)}\left(d_{\perp} L_{r}\right)^{\left[2\left(d_{\perp}-1\right)\right] /\left(d_{\perp}+2\right)}} \\
& \times\left[\frac{3 \Delta_{\mathcal{R}}^{2}(k)}{8 \beta\left(d_{\perp}\right) \alpha^{2}\left(r_{\|}\right)}\right]^{d_{\perp} /\left(d_{\perp}+2\right)}
\end{aligned}
$$

$$
\begin{aligned}
& \simeq \frac{0.025\left[A\left(k_{0}\right)\right]^{1 / 2}}{\left[25 \alpha\left(r_{\|}\right)\right](10 \theta)}\left(\frac{10}{\theta L_{r}}\right)^{1 / 2}\left(\zeta_{i} M_{s} r_{\perp}\right)^{2} \quad\left[d_{\perp}=2\right] \\
& \simeq \frac{0.025\left[A\left(k_{0}\right)\right]^{2 / 3}}{\left[25 \alpha\left(r_{\|}\right)\right]^{4 / 3}(10 \theta)}\left(\frac{10}{\theta L_{r}}\right)\left(\zeta_{i} M_{s} r_{\perp}\right)^{4} \quad\left[d_{\perp}=4\right],
\end{aligned}
$$

where we have let $y_{i}=2 \pi r_{\perp} \zeta_{i}$ with $\zeta_{i} \lesssim 1$. To get $L_{\text {inf }} \gtrsim 60$, we must require $\zeta_{i} M_{s} r_{\perp} \gtrsim 50$ for $d_{\perp}=2$ or $\zeta_{i} M_{s} r_{\perp} \gtrsim 10$ for $d_{\perp}=4$. Note, though, that for large $\theta$, it is not possible to have enough expansion during inflation. In this case, the images of one brane exert non-trivial forces on the other brane, resulting in a power-law type potential.

\section{B. Power law inflation}

Next, we consider potentials of the form

$$
V(\psi)=V_{0}\left(1-\eta \psi^{\sigma}\right)
$$

such potentials arise for a brane situated near the origin. The value of $\sigma$ depends on the compactification topology. For hypercubic compactification, $\sigma=4$, whereas in other cases, $\sigma=2$. Note that in actuality the potential need not depend just on interbrane separation in such a picture, and the trajectory of the brane can be complicated. Here, though, we confine ourselves to simple one dimensional (diagonal) brane motion.

Following Eq. (18), we see that the origin $-\psi=0$-is an unstable equilibrium point, and any perturbation away from it will result in slow motion of the brane. For $\sigma>2$, the slow roll solution is

$$
\begin{aligned}
\psi & =\left[\psi_{i}^{\sigma-2}-\sigma(\sigma-2) \eta M_{P}^{2} L\right]^{1 /(\sigma-2)} \\
& =\left[\sigma(\sigma-2) \eta M_{P}^{2} L_{r}\right]^{1 /(\sigma-2)}
\end{aligned}
$$

and the total number of $e$-folds in inflation is

$$
L_{\mathrm{inf}}=\frac{\psi_{i}^{\sigma-2}}{\sigma(\sigma-2) \eta M_{P}^{2}}
$$

where $\psi_{i}$ is the starting value for the inflaton. Quantum fluctuations will imply $\psi_{i}=\zeta_{i} H / 2 \pi$, where $\zeta_{1} \sim 1$. The curvature fluctuation spectrum is

$$
\Delta_{\mathcal{R}}^{2}(k)=\frac{V_{0}(\sigma-2)^{2}[\sigma(\sigma-2) \eta]^{2 /(\sigma-2)} M_{P}^{2(4-\sigma) /(\sigma-2)} L_{r}^{2(\sigma-1) /(\sigma-2)}}{12 \pi^{2}} .
$$


The implied fluctuation spectrum is acceptably flat:

$$
\begin{aligned}
n_{s}(k)-1 & =\frac{d \ln \Delta_{\mathcal{R}}^{2}(k)}{d \ln k} \\
& =-\frac{2(\sigma-1)}{(\sigma-2) L_{r}(k)} \simeq-\frac{0.03(\sigma-1)}{\sigma-2}\left[\frac{60}{L_{r}(k)}\right] \\
\frac{d n_{s}(k)}{d \ln k} & =-\frac{2(\sigma-1)}{L_{r}^{2}(k)(\sigma-2)} \\
& \simeq-6 \times 10^{-4}\left(\frac{\sigma-1}{\sigma-2}\right)\left[\frac{60}{L_{r}(k)}\right]^{2} .
\end{aligned}
$$

For $\sigma=4$, Eqs. (21) and (20) become

$$
\begin{aligned}
\Delta_{\mathcal{R}}^{2}(k) & =\frac{8 \eta V_{0} L_{r}^{3 / 2}}{3 \pi^{2}} \\
L_{\mathrm{inf}} & =\frac{\zeta_{i}^{2} V_{0}}{96 \pi^{2} M_{P}^{4}\left(\eta V_{0}\right)} ;
\end{aligned}
$$

the observational constraints on the curvature fluctuation spectrum therefore require

$$
\eta V_{0}=\frac{3 \pi^{2} \Delta_{\mathcal{R}}^{2}(k)}{8 L_{r}^{3 / 2}} \simeq 2.5 \times 10^{-11} A\left(k_{0}\right)\left(\frac{60}{L_{r}}\right)^{3 / 2}
$$

i.e., the potential must be extremely flat. This requirement is well known from studies of new inflation, which sometimes idealize the potential to Eq. (18) with a small dimensionless parameter $\lambda$ equivalent to $\eta V_{0}$. In Ref. [16], a particular toroidal compactification is proposed where this small parameter is ( $F$ is a geometrical factor related to the compactification geometry)

$$
\eta V_{0} \simeq \frac{g_{s} \theta^{4} F \beta}{16 \pi \alpha^{3}}\left(\frac{M_{s}}{M_{P}}\right)^{4},
$$

which can be small enough for $\theta \sim 0.1$ provided that

$$
\frac{M_{s}}{M_{P}} \simeq 10^{-3} .
$$

In this picture, the flatness of the effective potential is attributed to a relatively small value of the string scale compared with the Planck mass.

Special treatment is required for $\sigma=2$, which is expected for any non-hypercubic compactification topology. For this case, the scale factor grows like a power law in time during slow roll:

$$
\psi=\psi_{i}\left(\frac{a}{a_{i}}\right)^{2 \eta M_{P}^{2}}=\psi_{f}\left(\frac{a}{a_{f}}\right)^{2 \eta M_{P}^{2}}
$$

where $\psi_{f}$ and $a_{f}$ are the values of the field and scale factor at the end of slow rolling. Since $d \ln \psi / d \ln a=2 \eta M_{P}^{2}$, we require $\eta M_{P}^{2} \ll 1$ for slow rolling. It is easy to see that for this potential, $\ddot{\psi} / 3 H \dot{\psi}=2 \eta M_{P}^{2} / 3 \ll 1$. Slow roll ends, for this potential, only when $\dot{\psi}^{2} / 2 V_{0}=2 \eta^{2} M_{P}^{2} \psi^{2} / 3 \simeq 1$, or $\psi_{f}$ $\simeq\left(\eta M_{P}\right)^{-1} \gg M_{P}$, or when the polynomial approximation to the potential fails, which happens when the brane moves a substantial fraction of a lattice spacings. The total number of $e$-folds in inflation is

$$
L_{\mathrm{inf}}=\frac{\ln \left(\psi_{f} / \psi_{i}\right)}{2 \eta M_{P}^{2}}=\frac{\ln \left(y_{f} / y_{i}\right)}{2 \eta M_{P}^{2}} .
$$

The curvature fluctuation spectrum for this case is

$$
\begin{aligned}
\Delta_{\mathcal{R}}^{2}(k) & =\frac{\left(H / 2 \pi \psi_{f}\right)^{2} e^{4 \eta M_{P}^{2} L_{r}}}{4\left(\eta M_{P}^{2}\right)^{2}} \\
& =\frac{\left(H / 2 \pi \psi_{i}\right)^{2}\left(a_{i} / a\right)^{4} \eta M_{P}^{2}}{4\left(\eta M_{P}^{2}\right)^{2}} \\
& =\frac{\left(H / 2 \pi \psi_{i}\right)^{2}\left(a_{i} H / k\right)^{4} \eta M_{P}^{2}}{4\left(\eta M_{P}^{2}\right)^{2}},
\end{aligned}
$$

where evaluating at horizon crossing implies that $k=H a$, which has been used to get the final form of the spectrum. In this case,

$$
n_{s}-1=\frac{d \ln \Delta_{\mathcal{R}}^{2}(k)}{d \ln k}=-4 \eta M_{P}^{2}
$$

which is independent of $k$. The WMAP analysis implies that $\eta M_{P}^{2} \lesssim 0.01$. From the first form of Eq. (29), and $\eta M_{P}^{2}$ $\simeq 0.01$, it follows that the observed temperature fluctuations can be accounted for if $\left(V_{0}=2 \tau_{4} \ell_{\|}\right)$

$$
\frac{H}{2 \pi \psi_{f}} \sim\left(2 \pi M_{P} y_{f}\right)^{-1} \sim 10^{-7}\left(\frac{\eta M_{P}^{2}}{0.01}\right),
$$

in which case $L_{\mathrm{inf}} \sim 10^{3}$. For $y_{f} \simeq 2 \pi r_{\perp}$, this relation implies

$$
\frac{M_{s}}{M_{P}} \sim 10^{-6}\left(\frac{\eta M_{P}^{2}}{0.01}\right) M_{s} r_{\perp}
$$

which is generally smaller than our previous estimates unless $M_{s} r_{\perp} \sim 10^{3}$.

\section{Cosmic string properties}

Because the inflaton and the ground state open string modes responsible for defect formation are different, and the ground state open string modes become tachyonic and develop vacuum expectation values only towards the end of the inflationary epoch, various types of defects (lowerdimensional branes) may be formed. A priori, defect production after inflation may be a serious problem. Fortunately, it is argued in Refs. [16,17] that, from the properties of superstring/brane theory and the cosmological evolution of the universe, the only defects copiously produced are cosmic strings. In superstring theory, $\mathrm{D} p$ branes come with either odd $p$ (in Sec. II B theory) or even $p$ (in Sec. II A theory). 
The collision of a $\mathrm{D} p$ brane with another $\mathrm{D} p$ brane at an angle (or with an anti-D $p$-brane) yields $\mathrm{D}(p-2)$ solitons (i.e., codimension 2). Topologically, a variety of defects may be produced. Because they have even codimensions with respect to the branes that collide, they have specific properties [22]. Cosmologically, since the compactified dimensions tangent to the brane are smaller than the Hubble size, the Kibble mechanism works only if all the codimensions are tangent to the uncompactified dimensions. As a consequence, only cosmic strings may be copiously produced $[16,17]$.

The observational imprint of cosmic strings is determined primarily by the product of Newton's constant and the cosmic string tension $G \mu$ assuming the evolution of the string network can reach the scaling regime. The value of $\mu$ implied by superstring cosmology depends on several parameters, but is most sensitive to the string scale $M_{s}$. To get an order of magnitude estimate, we may use the small $\theta$ case, which is arguably the most likely inflationary scenario.

The cosmic strings may be D1 branes, but most likely, they are $\mathrm{D}(p-2)$-branes wrapping around $(p-3)$ cycles in the compactified dimensions. If the D1 brane is the cosmic string (i.e., $p=3$ ), its tension is simply the cosmic string tension

$$
\mu=\tau_{1}=\frac{M_{s}^{2}}{2 \pi g_{s}} .
$$

However, we expect the string coupling generically to be $g_{s} \gtrsim 1$. (It is well known that radion and dilaton moduli are not stabilized by perturbative dynamics in string theory. Presumably, any superstrongly coupled string model is dual to a weakly coupled one, and thus cannot stabilize the moduli either. We therefore expect a moderately strong string coupling, since only in this case we will find non-trivial dynamics.) To obtain a theory with a weakly coupled sector in the low energy effective field theory (i.e., the standard model of strong and electroweak interactions with weak gauge coupling constant $\alpha$ ), it then seems necessary to have the brane world picture, in which we have the $\mathrm{D} p$ branes for $p>3$, where the $(p-3)$ dimensions are compactified to volume $V_{\|}$. Now the cosmic strings are $\mathrm{D}(p-2)$ branes, with the $(p-3)$ dimensions compactified to the same volume $V_{\|}$. Noting that a D $p$-brane has tension $\tau_{p}=M_{s}^{p+1} /(2 \pi)^{p} g_{s}$, the tension of such cosmic strings is

$$
\mu=\frac{M_{s}^{p-1} V_{\|}}{(2 \pi)^{p-2} g_{s}}=\frac{M_{s}^{2} v_{\|}}{2 \pi g_{s}}=\frac{M_{s}^{2}}{4 \alpha \pi} \simeq 2 M_{s}^{2},
$$

for $\alpha \simeq \alpha_{\mathrm{GUT}} \simeq 1 / 25$. For one pair of branes at an angle $\theta$, only this type of cosmic strings is produced topologically. For a large enough stack of branes colliding, the D1 branes may also be allowed topologically, but they are not produced cosmologically. Thus, $\mu \simeq 2 M_{s}^{2}$ is a reasonably general estimate. We considered estimates of $M_{s}$ implied in various scenarios for brane inflation in Secs. II A and II B. These estimates are broadly consistent with

$$
10^{-6} \gtrsim G \mu \gtrsim 10^{-11},
$$

although a smaller range is obtained in any specific model or class of setups. For example, for branes colliding at a small angle, a likely range is

$$
5 \times 10^{-7} \gtrsim G \mu \gtrsim 7 \times 10^{-8} \text {. }
$$

Thus, brane inflation can lead to cosmic string tensions below, but not far below, current observational bounds.

\section{Tensor modes}

During slow roll, the tensor power is

$$
\Delta_{h}^{2}(k)=\frac{128 G^{2} V_{0}}{3}=\frac{2 V_{0}}{3 \pi^{2} M_{P}^{4}},
$$

and is smaller than the scalar power by the factor

$$
r(k)=8 M_{P}^{2}\left(\frac{V^{\prime}}{V}\right)^{2}=\frac{8}{M_{P}^{2}}\left(\frac{d \phi}{d \ln a}\right)^{2} .
$$

How small $r(k)$ is depends on the specific brane inflation model. For branes intersecting at an angle $\theta$ we find that

$$
\begin{aligned}
\Delta_{h}^{2}(k)= & \frac{2 V_{0}}{3 \pi^{2} M_{P}^{4}}=\frac{\theta^{2} M_{s}^{4}}{96 \pi^{5} \alpha\left(r_{\|}\right) M_{P}^{4}} \\
= & 3.3 \times 10^{-12}(10 \theta)^{2}\left[25 \alpha\left(r_{\|}\right)\right]\left[\frac{10 A\left(k_{0}\right)}{\theta L_{r}}\right] \quad\left[d_{\perp}=2\right] \\
= & 5.4 \times 10^{-17}(10 \theta)^{2}\left[25 \alpha\left(r_{\|}\right)\right]^{1 / 3}\left[A\left(k_{0}\right)\right]^{4 / 3}\left(\frac{10}{\theta L_{r}}\right)^{2} \\
& {\left[d_{\perp}=4\right] . }
\end{aligned}
$$

In this case, the amplitude of the scalar mode is smaller than the amplitude of the perturbations due to cosmic strings by a small numerical factor times $\theta^{2}$, unless cosmic string intercommutation is extremely inefficient; see Secs. II C and III below. For power law brane-antibrane inflation, $\theta \rightarrow \pi$, and $V_{0}=M_{s}^{4} /(2 \pi)^{3} \alpha[13,16]$, so for this case we find

$$
\Delta_{h}^{2}(k)=\frac{M_{s}^{4}}{12 \pi^{5} \alpha M_{P}^{4}} .
$$

Nominally, these perturbations can be comparable to those induced by cosmic strings, although they may be relatively suppressed by the small numerical factor $\left(12 \pi^{5} \alpha\right)^{-1}$ $\simeq 0.007 /(25 \alpha)$. However, the spectrum of fluctuations produced by cosmic strings will still distinguish them from those due to primordial tensor modes. Both strings and the primordial tensor modes result in the $B$-type polarization of the cosmic microwave background radiation (CMBR). The predicted angular power spectrum $C_{l}^{B B}$ has been calculated for tensor modes from inflation (see, e.g., [23]). It has a generic feature that most of the power is on larger angular scales, in the region $l \lesssim 100$. This is very different from the shape of the $C_{l}^{B B}$ spectrum predicted by cosmic strings. There the dominant contribution comes from the vector 
modes and, as one can see from Fig. 4, most of the power is on smaller scales: $700 \leqq l \leqq 1000$.

As of today, the $B$-type polarization has not been detected [24] and the experimental constraint on $r(k)$ is rather mild: $r\left(k_{0}=0.002 \mathrm{Mpc}^{-1}\right) \leq 0.71[7]$.

\section{CMB, MATTER DENSITY AND POLARIZATION POWER SPECTRA: CALCULATION AND INTERPRETATION}

The fluctuations expected to arise from brane inflation should be an incoherent superposition of contributions from adiabatic perturbations initiated by curvature fluctuations $\Delta_{\mathcal{R}}^{2}(k)$ and active perturbations induced by the decaying cosmic string network. For example, the resulting CMB temperature maps will yield

$$
C_{l}=W C_{l}^{\text {adiabatic }}+B C_{l}^{\text {strings }},
$$

where $W$ and $B$ are weighting factors. Analogous expressions hold for matter density and polarization power spectra. In Eq. (41), the weight factors $W$ and $B$ determine the relative importance of the adiabatic and cosmic string contributions. We choose the weight factor $W$ so that $W=1$ when there are no cosmic strings.

In computing the combined effects of adiabatic and cosmic string perturbations, we have kept the cosmological background parameters fixed at their best-fit values according to [5]. In addition to $B$ and $W$, we vary the spectral index of the scalar curvature fluctuations, $n_{s}$. The tensor contribution to the adiabatic component was set to zero, since, as discussed in Sec. II D, it is likely to be small. When fitting to both WMAP and the 2dFGRS data, we considered two cases (described in more detail in Sec. III B): with bias $b$ fixed and with $b$ being an additional parameter of the fit.

\section{A. Cosmic strings and the CMBR}

Perturbations due to cosmic strings were calculated using the model first introduced in [9] and further developed in $[25,26]$. The main idea is to represent the cosmic string network by a collection of uncorrelated, straight string segments moving with random, uncorrelated velocities. All segments are produced at some early epoch and, at every subsequent epoch, a certain fraction of the number of segments decays in a way that maintains network scaling. The length of each segment at any time is taken to be equal to the correlation length of the network which, together with the root mean square velocity of segments, are computed from the velocitydependent one-scale model of Martins and Shellard [27]. The positions of segments are drawn from a uniform distribution in space and their orientations are chosen from a uniform distribution on a two sphere.

This model is a rather crude approximation of a realistic string network. However, with a suitable choice of model parameters, its main predictions for $\mathrm{CMB}$ and matter power spectra have been shown to be in agreement with results obtained using other local string sources [28,29]. The main advantage of our model is its flexibility. For example, parameters can be chosen to describe strings with different scaling properties, different amounts of small scale structure etc. This is especially valuable when describing strings produced in brane inflation, since strings are expected to intercommute with a lower probability in the presence of extra spatial dimensions [18].

It is well known that properties and possible observational signatures of global and local strings can be dramatically different. Global strings predict almost no power on small angular scales for the CMB temperature anisotropy [30], while local strings produce a quite significant broad peak at $l \sim 450$ in a spatially flat universe $[9,25,28,29,31]$. Also, global strings induce a significantly larger vector component of metric perturbations. Consequently, their prediction for the strength of the $B$-type polarization [32] is generally higher than that of local strings.

The perturbations due to cosmic strings are proportional to the $G \rho_{s}$, where $\rho_{s}$ is their mass density. In a model where the string network has a single characteristic scale $L(t)$ at time $t$, the density $\rho_{s}=\mu L / L^{3}=\mu / L^{2}$ evolves according to

$$
\dot{\rho}_{s}+2 H \rho_{s}=-\frac{\lambda \rho_{s}}{L}=\frac{\lambda \rho_{s}^{3 / 2}}{\mu^{1 / 2}},
$$

where the parameter $\lambda$ governs the probability of intercommutation of the strings. Equation (42) has a scaling solution,

$$
\rho_{s}=\frac{4 \mu}{\lambda^{2}}\left(a(t) \int^{t} \frac{d t^{\prime}}{a\left(t^{\prime}\right)}\right)^{-2}
$$

after transient effects die away. Since the strings that arise in brane inflation reside in $\left(4+d_{\perp}\right)$ dimensions, the value of $\lambda$ is substantially reduced, and one might think that $B$ $\propto\left(G \mu / \lambda^{2}\right)^{2}$, which would mean that even if WMAP would allow a moderately large value of $B$, the implied value of $G \mu$ would actually be rather small.

In fact, the scaling of $B$ with $\lambda$ is not quite this severe. Consider a string network in a volume $V$ described by the one-scale model. Let the characteristic scale at some time be $L(t)$. This assumes that on an average there is one string segment of length $L$ per volume $L^{3}$. The rough number of such string segments is

$$
N=\frac{V}{L^{3}}
$$

If the energy per unit length is $\mu$, then the total energy of the string network is

$$
E=N \mu L=\frac{V \mu}{L^{2}},
$$

and the energy density is just $\rho=E / V=\mu / L^{2}$.

Now suppose we want to calculate the effect of this string network on the CMB temperature anisotropy. In particular, we want to find the power spectrum, i.e., the 2-point function. For simplicity, let us assume that only $\rho(t)$ affects CMB (in general we would have to consider all components of the string network's energy-momentum tensor $T_{\mu}^{\nu}$ ). Then to evaluate the CMB power spectrum it suffices to know the 
2-point unequal time correlators $\left\langle\rho\left(k, t_{1}\right) \rho\left(k, t_{2}\right)\right\rangle$ at all $k, t_{1}$ and $t_{2}$. Here $\rho(\mathbf{k}, t)$ is the Fourier transform of $\rho(\mathbf{x}, t)$. The $\mathrm{CMB}$ power spectrum is roughly given by

$$
C_{l}=\int d k \int d t_{1} \int d t_{2} \hat{L}_{l}\left(t_{1}, t_{2}, k\right)\left[\left\langle\rho\left(k, t_{1}\right) \rho\left(k, t_{2}\right)\right\rangle\right],
$$

where $\hat{L}_{l}\left(t_{1}, t_{2}, k\right)$ is a linear operator.

Again, for simplicity, let us assume that $L=\xi t$ at all times, namely that the network scales perfectly with time. We want to see how $C_{l}$, or equivalently $\left\langle\rho\left(k, t_{1}\right) \rho\left(k, t_{2}\right)\right\rangle$, depends on $\xi$ and $\mu$. Let us also assume that the segments are straight.

At time $t$ there are roughly $N(t)=V / L^{3}(t)$ segments and

$$
\rho(k, t)=\sum_{i=1}^{N(t)} \rho^{(i)}(k, t) \equiv \sum_{i=1}^{N(t)} \tilde{\rho}^{(i)}(k, t) \mu L(t),
$$

where $\tilde{\rho}^{(i)}(k, t)=\rho^{(i)}(k, t) /[\mu L(t)]$ to factor out dependences on $\mu$ and $L(t)$ [the phase of $\tilde{\rho}$ may still depend on $L(t)$ but the amplitude does not]. Now we can write

$$
\begin{aligned}
& \left\langle\rho\left(k, t_{1}\right) \rho\left(k, t_{2}\right)\right\rangle \\
& \quad=\sum_{i=1}^{N\left(t_{1}\right)} \sum_{j=1}^{N\left(t_{2}\right)}\left\langle\tilde{\rho}^{(i)}\left(k, t_{1}\right) \rho^{(j)}\left(k, t_{2}\right)\right\rangle \mu^{2} L\left(t_{1}\right) L\left(t_{2}\right) .
\end{aligned}
$$

Because individual segments are statistically independent

$$
\left\langle\widetilde{\rho}^{(i)}\left(k, t_{1}\right) \rho^{(j)}\left(k, t_{2}\right)\right\rangle=\delta_{i j}\left\langle\tilde{\rho}^{(i)}\left(k, t_{1}\right) \tilde{\rho}^{(i)}\left(k, t_{2}\right)\right\rangle,
$$

and therefore

$$
\begin{aligned}
& \left\langle\rho\left(k, t_{1}\right) \rho\left(k, t_{2}\right)\right\rangle \\
& \quad=\sum_{i=1}^{\min \left[N\left(t_{1}\right), N\left(t_{2}\right)\right]}\left\langle\tilde{\rho}^{(i)}\left(k, t_{1}\right) \tilde{\rho}^{(i)}\left(k, t_{2}\right)\right\rangle \mu^{2} L\left(t_{1}\right) L\left(t_{2}\right) .
\end{aligned}
$$

To interpret $\min \left[N\left(t_{1}\right), N\left(t_{2}\right)\right]$ it might help to think that all segments were there at the initial time but over course of their evolution some of them decayed. For certainty, let us assume $t_{1}<t_{2}$ and therefore $N\left(t_{1}\right)>N\left(t_{2}\right)$. We can now write

$$
\begin{aligned}
\left\langle\rho\left(k, t_{1}\right) \rho\left(k, t_{2}\right)\right\rangle & =\sum_{i=1}^{N\left(t_{2}\right)}\left\langle\widetilde{\rho}^{(i)}\left(k, t_{1}\right) \tilde{\rho}^{(i)}\left(k, t_{2}\right)\right\rangle \mu^{2} L\left(t_{1}\right) L\left(t_{2}\right) \\
& =N\left(t_{2}\right)\left\langle\left[\tilde{\rho}^{(1)}\left(k, t_{2}\right)\right]^{2}\right\rangle \mu^{2} L\left(t_{1}\right) L\left(t_{2}\right),
\end{aligned}
$$

where the last step is possible because all segments are statistically identical and hence

$$
\left\langle\tilde{\rho}^{(1)}\left(k, t_{1}\right) \tilde{\rho}^{(1)}\left(k, t_{2}\right)\right\rangle=\left\langle\tilde{\rho}^{(i)}\left(k, t_{1}\right) \tilde{\rho}^{(i)}\left(k, t_{2}\right)\right\rangle \text { for all } i .
$$

Substituting $L=\xi t$ and $N(t)=V /[L(t)]^{3}$ we find

$$
\begin{aligned}
\left\langle\rho\left(k, t_{1}\right) \rho\left(k, t_{2}\right)\right\rangle & =\frac{V}{\xi^{3} t_{2}^{3}} \mu^{2} \xi^{2} t_{1} t_{2}\left\langle\tilde{\rho}^{(1)}\left(k, t_{1}\right) \tilde{\rho}^{(1)}\left(k, t_{2}\right)\right\rangle \\
& =\frac{\mu^{2}}{\xi} \times F\left(k, t_{1}, t_{2}\right)
\end{aligned}
$$

where $F\left(k, t_{1}, t_{2}\right)$ is independent of $\xi$ or $\mu$, and therefore

$$
C_{l}^{\text {strings }} \propto \frac{\mu^{2}}{\xi} .
$$

In one-scale model parameter $\xi$ is usually directly proportional to $\lambda$, and this is just what is found numerically. Correspondingly, the meaning of the parameter $B$ is

$$
B=\frac{C_{l}(\lambda, G \mu)}{C_{l}\left(\lambda_{0}, G \mu_{0}\right)}=\frac{\mu^{2}}{\mu_{0}^{2}} \frac{\lambda_{0}}{\lambda},
$$

with $G \mu_{0}=2 \times 10^{-6}$ and $\lambda_{0} \sim 0.25$ adopted as reference values.

Thus, it follows that $C_{l} \propto \lambda_{0} / \lambda$ and not $\left(\lambda_{0} / \lambda\right)^{4}$. The reason is that the network is random on scales larger than $L$ and two-point functions vanish for contributions from uncorrelated parts of the network. In practice (as we have numerically verified), Eq. (55) does not hold exactly for all $l$ for temperature $C_{l}$, s, because of other effects taking place. It is different for scalar, tensor and vector parts (variations are up to $50 \%$ ). However, Eq. (55) is nearly exact for the quadrupole and hence almost exact for all polarization spectra, since their source is the temperature quadrupole.

\section{B. Methods}

We have performed a partial statistical analysis in which we held the parameters of the background cosmological model (total, matter, baryon and dark energy density parameters, Hubble constant, reionization optical depth) fixed at their WMAP best fit values according to [5]. More specifically, we considered a flat $\Lambda \mathrm{CDM}$ universe with $\Omega_{\mathrm{CDM}}$ $=0.225, \Omega_{b}=0.045, \Omega_{\Lambda}=0.73, H_{0}=71 \mathrm{~km} / \mathrm{s} / \mathrm{Mpc}$ and $\tau$ $=0.17$. The scalar spectral index $n_{s}$ was allowed to vary within bounds set by the prior $0.8 \leqslant n_{s} \leqslant 1.2$, in increments of $\Delta n_{s}=1.25 \times 10^{-3}$.

The string spectra were calculated only once, using the string model parameters chosen to produce spectra that roughly agree with $[9,25,28,29]$. In particular, we set $G \mu_{0}$ $=2 \times 10^{-6}$, which, if strings were the only source of inhomogeneity, would result in temperature anisotropy in a rough agreement with observations on Cosmic Background Explorer (COBE) scales. The string intercommutation probability $\lambda$ was $\sim 0.25$ and was allowed to vary only insignificantly during the radiation-matter domination transition.

The CMBR and linear matter power spectra for both adiabatic and string parts were computed using, respectively, modified versions of CMBFAST [33]. However, one cannot directly compare linear matter spectra outputted by CMBFAST to the galaxy clustering data published by the 2dFGRS team. We have "processed" the theoretical power spectrum $P^{t h}(k)$ for both adiabatic and string components following a proce- 
dure similar to that prescribed in Sec. 5.1.4 of [6]. First of all, we output $P^{\text {th }}(k)$ at the effective redshift of the 2dFGRS survey: $z_{\text {eff }}=0.17$ (the valued suggested in [6]). Then we correct for the redshift space distortions using the approximate formula given in [6]:

$$
P^{t h}(k) \rightarrow P_{z}^{t h}(k)=\left(1+\frac{2}{3} \beta+\frac{1}{5} \beta^{2}\right) P^{t h}(k),
$$

with $\beta=0.45$. We then convolve it with the $2 \mathrm{dF}$ window function [19] using the matrix $M_{W}$ provided in Ref. [42]:

$$
P_{z}^{t h}(k) \rightarrow P_{W}(k)=\sum_{q} M_{W}(k, q) P_{z}^{t h}(q),
$$

and, finally, multiply by the bias factor to obtain the spectrum that can be compared to data:

$$
P(k)=b^{2} P_{W}(k),
$$

where $b=\Omega_{m}^{0.6} / \beta$.

We have chosen to fit to the binned version of WMAP data, for both temperature (TT) and cross temperaturepolarization (TE) angular spectra. When fitting to WMAP $+2 \mathrm{dF}$ we had a choice of making the bias $b$ an additional parameter or using a prescribed fixed value. We have investigated both possibilities and will refer to them the " $b$-free" and " $b$-fixed" model.

\section{Results}

We are interested in constraining the fractional contribution to the WMAP and 2dFGRS data given by Eq. (41). To do this properly, from first principles, would require using theoretical models with both cosmic strings and adiabatic perturbations to generate the relevant sky maps, and then compare these directly to the data to compute likelihood functions that can be used, along with appropriate priors, the posterior probability distributions for the parameters of the models. To get a quick and dirty bound on the most interesting parameter $B$ we have chosen to adopt a simpler approach in which we treat the published results for $C_{l}$ of the TT and TE sky maps, and the $2 \mathrm{dFGRS}$ power spectra as data, and use them and their uncertainties to construct a $\chi^{2}$ statistic in the usual way. For each value of $B$, we minimize the statistic with respect to variations in the parameters $n_{s}, W$, and the bias parameter, to find $\chi_{\min }^{2}(B)$.

If our "data" really represented independent data points, then we could be confident that at each $B$ the value of $\chi_{\min }^{2}(B)$ would obey a distribution that roughly has a mean value equal to the number of degrees of freedom in the fit, $\nu$, and a standard deviation $\sqrt{2 \nu}$. Figure 1 shows the results for $b$ free (top) and $b$ fixed (bottom) (as defined at the end of Sec. III B). For our procedure, we have $N=38$ (TT) $+26(\mathrm{TE})+32(2 \mathrm{dF})=96$ data points. We keep cosmological paramters such as $\Omega_{m}, \Omega_{b}, \Omega_{\Lambda}, h$ and $\tau$ (reionization) fixed at their best fit values from [5]. Therefore, we have either three $\left(n_{s}, W\right.$, and $b$ for $b$-free evaluation) or two $\left(n_{s}\right.$ and $W$ for $b$-fixed evaluation) parameters in each of the panels in
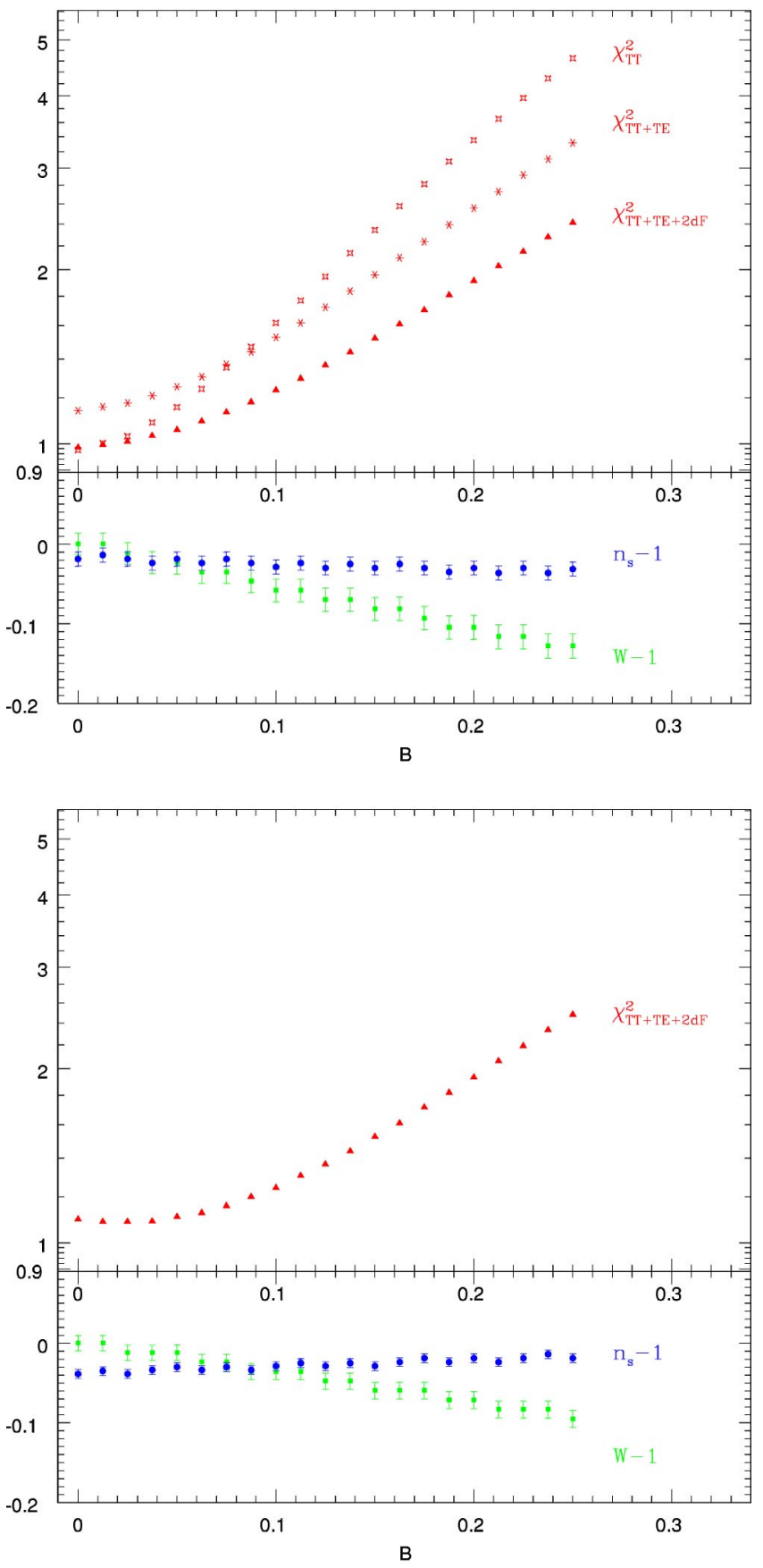

FIG. 1. (Top) Minimum reduced $\chi^{2} / \nu$ as a function of $B$ for the fit of the $b$-free model to the WMAP's TT (triangles), TT+ TE (stars) and $\mathrm{TT}+\mathrm{TE}+2 \mathrm{dF}$ (hollow squares) with corresponding best fit values of $W-1$ (squares) and $n_{s}-1$ (circles). We only show $W$ and $n_{s}$ from the fit to the TT data alone. The error bars correspond to the diagonal elements of the covariance matrix. Adding the remaining datasets changes the best fit values of $W$ and $n_{s}$ by only a small amount, well within the plotted error bar. (Bottom) Same as on the top but for the $b$-fixed model with all datasets included. In the top panel, $\nu=93$ and in the bottom $\nu=94$. The values of the reduced $\chi^{2} / \nu$ at $B=0$ are, in the top panel, 0.97 (TT), 1.1 (TT $+\mathrm{TE})$ and $0.98(\mathrm{TT}+\mathrm{TE}+2 \mathrm{dF})$, and 1.1 in the bottom panel. 
Fig. 1, so $\nu=93$ ( $b$ free) or 94 ( $b$ fixed), and $\sqrt{2 \nu} \simeq 14$ in either case. Since the two panels in the figure actually show the reduced statistic $\chi^{2}(B) / \nu$, we could regard values of $B$ with $\chi^{2}(B) / \nu$ within $\approx 0.15 n$ of the minimum value as representing more or less equally good fits to the data to within $n$ "sigma." Below, we shall use $\chi^{2} / \nu \lesssim 1.25$ for placing a limit on $B$; this can be interpreted as either a 1.7 " $\sigma$ " bound, or as a crude attempt to account for the fact that we are also ignoring the effects of varying several more cosmological parameters. Although assessing the acceptability of models with different values of $B$ is not justified rigorously, we note that the minimum values of $\chi^{2} / \nu$ shown in either panel of Fig. 1 are close to one, which may be taken as a posteriori assurance that our method for comparing models with different values of $B$ may not be far off.

The top panel of Fig. 1, corresponding to the $b$-free model, contains plots of the minimized reduced $\chi_{\mathrm{TT}}^{2} / \nu$, $\chi_{\mathrm{TT}+\mathrm{TE}}^{2} / \nu$ and $\chi_{\mathrm{TT}+\mathrm{TE}+2 \mathrm{dF} /}^{2} \nu(\nu=93)$ as functions of $B$ computed using, respectively, WMAP's TT spectrum only, TT and TE, and TT, TE and the $2 \mathrm{dF}$ spectrum. As one can see from that plot, in all cases the lowest value of the minimum $\chi^{2}(B) / \nu$ occurs at $B=0$, i.e., when there is no contribution from strings. However, as additional datasets are added to the fit, the values of the minimum $\chi^{2}(B)$ become smaller. Figure 1 also shows the values of $n_{s}-1$ and $W-1$ values that minimized $\chi^{2} / \nu$ at each $B$, with estimated uncertainties, of the order of \pm 0.01 for both. Not plotted are the values of the bias parameter $b$, which varied from 0.97 at $B=0$ to 1.02 at $B=0.25$. Note that the value of $n_{s}-1$ is typically around $-0.02 \pm 0.01$ for $B \leqq 0.1$, the region where the minimum $\chi^{2}(B) / \nu$ is less than about 1.25 .

The bottom panel of Fig. 1 corresponds to the $b$-fixed model, i.e., the model in which the value of the bias was held fixed at $b=\Omega_{m}^{0.6} / \beta$. Results are shown for the minimum value of $\chi^{2}(B) / \nu$ constructed using all three datasets. Here we find that the lowest value of the minimum $\chi^{2}(B) / \nu$ is at $B=0.025$, not $B=0$. In view of the limitations of our statistical analysis, one cannot attach much significance to either the existence of this minimum, or the precise nonzero $B$ at which it occurs, but it is worth observing that adding a small string contribution may be welcome when matching the CMBR normalized matter spectra to the galaxy data. Moreover, this result shows that at the very least we cannot exclude values of $B \sim 0.025$. We note that the values of $n_{s}-1$ corresponding to the minimum $\chi^{2}$ per degree of freedom grow with $B$ in this panel and, for $B \leqq 0.1$, they fall within the range $n_{s}-1=-0.03 \pm 0.01$.

In Figs. 2 and 3 we plot the adiabatic plus string CMB and matter power spectra superposed using Eq. (41) with different values of $B$ and corresponding best fit values of $n_{s}$ and $W$ for the $b$-free and $b$-fixed models. In both figures, the solid line corresponds to $B=0$ and the dash-dot line to $(W=0, B=1)$.

The simple adiabatic $\Lambda \mathrm{CDM}$ model with a constant $n_{s}$ fits the WMAP data very well, in general, but much worse so on very large scales. For the first few multipoles, $2 \leqslant l \leqslant 6$, the experiment finds a clear deficit of power, as compared to the prediction of the model. One could hope that, because cos-
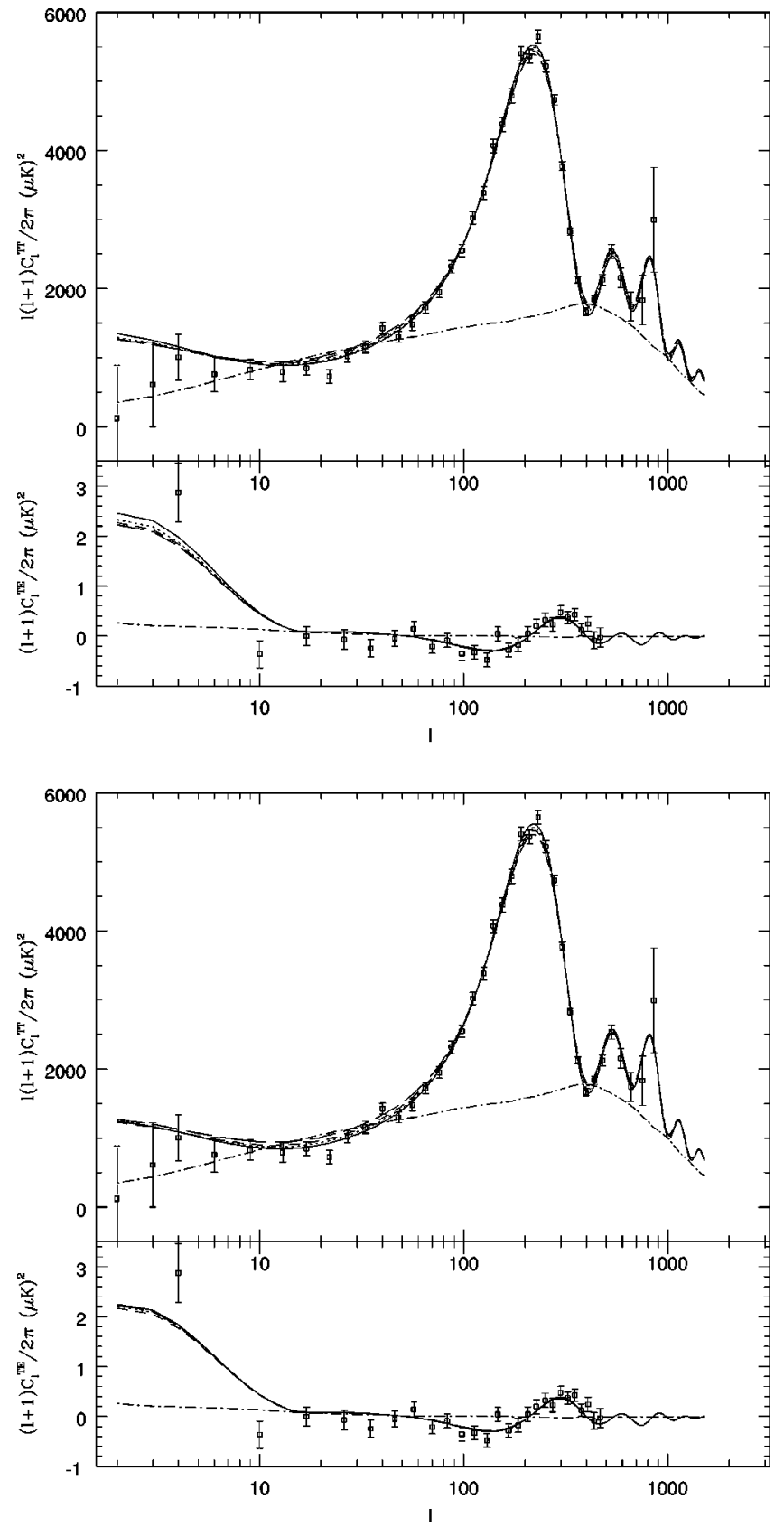

FIG. 2. (Top) Plots of the best fit $C_{l}^{\mathrm{TT}}$ and $C_{l}^{\mathrm{TE}}$ computed using the $b$-free model for different values of $B$ together with the WMAP's binned data. (Bottom) Corresponding plots of the galaxy clustering power spectra together with the 2dFGRS data. On both plots, the solid line corresponds to $B=0$, dotted line $-B=0.05$, short dash line $-B=0.1$, long dash line $-B=0.15$ and the dash-dot line corresponds to the pure string contribution, i.e., $(W=0, B$ $=1$ ).

mic strings generically predict such lack of power for low multipoles, adding a string component would improve the fit on large scales. However, as one can see from Figs. 2 and 3, this is not the case. The reason is that cosmic strings fit the data extremely poorly in the region of the first and the second acoustic peaks, where the WMAP error bars on $C_{l} \mathrm{~s}$ are very small. The "benefit" from adding strings on larger scales, where the error bars are large, is offset by the much 

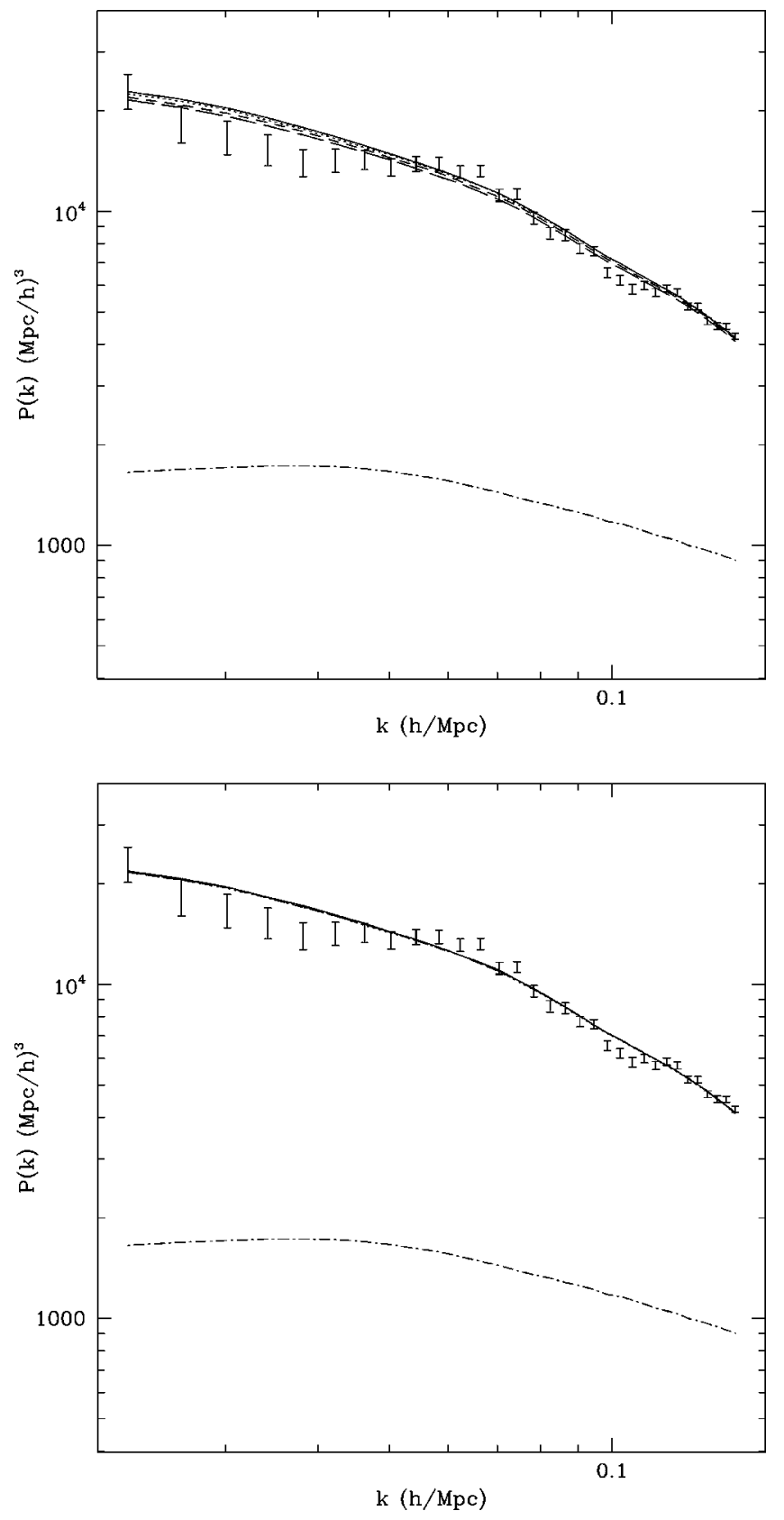

FIG. 3. Same as in Fig. 2 but for the $b$-fixed model.

larger "damage" that strings cause when fitting on smaller scales.

Clearly, our partial statistical analysis does not yield a rigorous bound on the magnitude of $B$. However, the results in Fig. 1 can be used to get a rough estimate for the allowed range of the $B$ values. From the discussion at the start of this section, a reasonable bound can be based on taking $\chi^{2}(B) / \chi^{2}(0) \lesssim 1.25$. In Fig. 1, the maximum value of $B$ corresponding to this criterion would be $B_{\text {max }} \sim 0.1$. Moreover, we saw that the actual minimum value of $\chi^{2}(B)$ in the bottom panel of Fig. 1 was at $B \simeq 0.025$, not zero, which indicates that, almost certainly, the available data cannot exclude values of $B \leqq 0.025$. As discussed in Sec. III $A$, this does not simply translate into a constraint on the value of the string tension, $G \mu$. Instead, from Eq. (55), $B \propto(G \mu)^{2} / \lambda$.

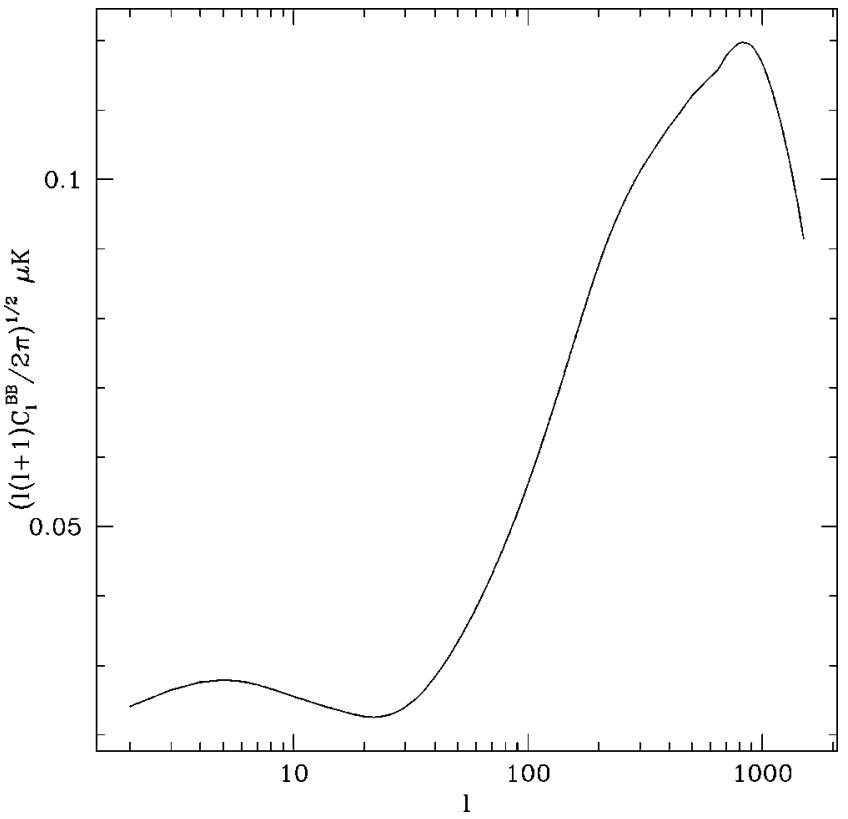

FIG. 4. The $B$-type polarization spectrum $C_{l}^{B B}$ due to cosmic strings as predicted by our string model with the fit parameter $B$ set to 0.1. Note that the $y$ axis has units of microkelvin and not (microkelvin) $^{2}$ as on the other $C_{l}$ plots.

Because cosmic strings produce a sizable vector perturbation, in addition to the $E$ polarization, they can also induce the $B$-type polarization of the CMBR. In Fig. 4 we plot the $B$-polarization angular spectrum $C_{l}^{B B}$ as predicted by our model with $B=0.1$. Experimentally, it would be extremely challenging to detect a $0.1 \mu \mathrm{K}$ polarization signal on scales $l \sim 800$, in large part because of lensing by galaxies and other systematic effects [34]. However, such a detection would be an important test for the existence of cosmic strings, and the possibility of accomplishing it is not ruled out.

\section{DISCUSSION}

Cosmic strings appear to be likely a by-product of inflation in superstring cosmology. Although these cosmological models are only just beginning to be developed in sufficient detail for comparisons with data to be possible, most models seem to feature a string scale comparable to or slightly smaller than the grand unified theory (GUT) scale, and cosmic string tensions $G \mu \sim 10^{-10}-10^{-6}$.

These expectations motivate studying models in which CMB fluctuations and large scale structure are the consequence of both growing modes associated with perturbations generated from quantum fluctuations during inflation, and active perturbations associated with the gravitational effects of a network of cosmic strings. Here, we have presented an attempt at such an analysis, based on a rough comparison of theoretical models with both adiabatic perturbations and cosmic strings with WMAP TT and TE power spectra as well as the $2 \mathrm{dFGRS}$ results on galaxy correlation functions. Our results need to be improved in various ways, both by allowing numerous cosmological parameters we held fixed to vary, 
and by computing likelihood functions, rather than using a rough criterion based on a reduced $\chi^{2}$ statistic. Nevertheless, they already indicate that although the data currently available generally favor models without cosmic strings, they may not exclude nonzero cosmic string tension and density definitively, provided the growth of fluctuations in the universe is not dominated by cosmic strings. Rather conservatively, we have argued that values of $B \leqq 0.1$ are not excluded, although this result needs to be put on firmer footing statistically; we are quite confident that $B \leqq 0.025$ cannot be excluded as yet. Since $B \simeq(0.25 / \lambda)\left(G \mu / 2 \times 10^{-6}\right)^{2}$, these values of $B$ would correspond to

$$
G \mu \lesssim 1.3 \times 10^{-6} \sqrt{\frac{B \lambda}{0.1}} .
$$

We have also found the values of $n_{s}-1$ that minimze the reduced $\chi^{2}$ statistic for different values of $B$. Conservatively, for the range $B \leqq 0.1$, the various different comparisons with data in the two panels of Fig. 1 are consistent with $n_{s}-1$ $\simeq-(0.04-0.01)$. Comparing with the predictions of various brane inflation models via Eqs. (9), (22) and (30), we note that the values we find are in agreement and certainly not in conflict with any of the models. However, we note that the Coulombic inflation models generally predict $\left|n_{s}-1\right|$ $\vdots-0.03$, at the low end of the range of values we infer from comparing with the data, while the power law models allow larger $\left|n_{s}-1\right|$; Eq. (22) implies $\left|n_{s}-1\right| \gtrsim 0.03$ for any value of $\sigma$ and Eq. (30) implies a constant power law index whose value depends on parameters of the brane inflation model. If $n_{s}(k)$ can be pinned down with greater precision, it may become possible to discriminate among different brane inflation models. We caution, though, since we have not varied the background cosmological model in obtaining these bounds, we cannot rule out that a more complete analysis would allow still larger values of $G \mu$, with other values of $n_{s}-1$. In a future publication we intend to present results of a more comprehensive study which would include varying all cosmological parameters as well as the relevant parameters of the string model, including their specific predictions for $n_{s}(k)$, and a better justified statistical analysis based on codes made available by the WMAP team for computing likelihood functions for any model for the production of temperature pertubations [6]. (Some modifications will be needed to account for the non-Gaussianity of the cosmic string perturbations.) In addition to the WMAP and the $2 \mathrm{dF}$ GRS data, the analysis will also include the latest results from the sload digital sky survey [35].

The key to our analysis is the idea that the universe is a patchwork quilt, with a little bit of cosmic strings thrown in to complicate the models. A smoking gun for the existence of cosmic strings could be the detection of $B$-mode polarization on smaller scales at an amplitude considerably larger than is predicted in inflation models without cosmic strings. Another potentially distinguishing signature of cosmic strings could be the detection of cosmological non-Gaussianity. Tests of the WMAP data [8] have so far been limited to constraining the type of non-Gaussianity expected from inflationary models. Some of the most commonly used tests of nonGaussianity, such as the bispectrum test, had actually been shown to be insensitive to possible contributions from cosmic strings [26]. Specially tailored tests are likely to be needed to detect string induced non-Gaussianity [36]. Cosmic strings may also be detected from the observation of identical galaxy pairs in close proximity on the sky [37]. Gravitational radiation from kinks in cosmic strings may also be detectable down to exceptionally small values of $G \mu$ [38]. Pulsar timing is likely to push bounds on the density in long wavelength gravitational radiation backgrounds [39] down by an order of magnitude or so, corresponding to a factor of 3 or so in $G \mu / \lambda^{2}$ [40], but a substantial contribution to the background from supermassive black hole binaries [41] may frustrate our ability to use these observations to constrain the cosmic string tension much further.

\section{ACKNOWLEDGMENTS}

We thank Richard Battye, Nick Jones, Will Kinney, Horace Stoica, Alex Vilenkin, and Neal Weiner for discussions, and Tom Loredo and Saul Teukolsky for critical comments. This research is partially supported by NSF (S.-H.H.T.), NASA (I.W.), and PPARC (L.P.). M.W. is supported by the NSF. I.W. acknowledges the hospitality of KITP, which is supported by NSF Grant PHY99-07949, where part of this research was carried out.
[1] G.F. Smoot et al., Astrophys. J. Lett. 396, L1 (1992); C.L. Bennett et al., ibid. 464, L1 (1996).

[2] MAXIMA-1 Collaboration, A.T. Lee et al., Astrophys. J. Lett. 561, L1 (2001); BOOMERANG Collaboration, C.B. Netterfield et al., Astrophys. J. 571, 604 (2002); DASI Collaboration, C. Pryke et al., ibid. 568, 46 (2002).

[3] A.H. Guth, Phys. Rev. D 23, 347 (1981); A.D. Linde, Phys. Lett. 108B, 389 (1982); A. Albrecht and P.J. Steinhardt, Phys. Rev. Lett. 48, 1220 (1982).

[4] C.L. Bennett et al., astro-ph/0302207.

[5] D.N. Spergel et al., astro-ph/0302209.

[6] L. Verde et al., astro-ph/0302218.

[7] H.V. Peiris et al., astro-ph/0302225.
[8] E. Komatsu et al., astro-ph/0302223.

[9] A. Albrecht, R.A. Battye, and J. Robinson, Phys. Rev. Lett. 79, 4736 (1997); Phys. Rev. D 59, 023508 (1999).

[10] F.R. Bouchet, P. Peter, A. Riazuelo, and M. Sakellariadou, Phys. Rev. D 65, 021301(R) (2002).

[11] L. Kofman, A. Linde, and A.A. Starobinsky, Phys. Rev. Lett. 76, 1011 (1996); I. Tkachev, S. Khlebnikov, L. Kofman, and A. Linde, Phys. Lett. B 440, 262 (1998); C. Contaldi, M. Hindmarsh, and J. Magueijo, Phys. Rev. Lett. 82, 2034 (1999); R.A. Battye and J. Weller, Phys. Rev. D 61, 043501 (2000); J. Yokoyama, Phys. Lett. B 212, 272 (1988); Phys. Rev. Lett. 63, 712 (1989)

[12] G. Dvali and S.-H.H. Tye, Phys. Lett. B 450, 72 (1999).

[13] C.P. Burgess, M. Majumdar, D. Nolte, F. Quevedo, G. Rajesh, 
and R.J. Zhang, J. High Energy Phys. 07, 047 (2001).

[14] J. Garcia-Bellido, R. Rabadán, and F. Zamora, J. High Energy Phys. 01, 036 (2002); M. Gomez-Reino and I. Zavala, ibid. 09, 020 (2002); C. Herdeiro, S. Hirano, and R. Kallosh, ibid. 12, 027 (2001).

[15] G. Dvali, Q. Shafi, and S. Solganik, "D-Brane Inflation," hep-th/0105203; K. Dasgupta, C. Herdeiro, S. Hirano, and R. Kallosh, Phys. Rev. D 65, 126002 (2002).

[16] N. Jones, H. Stoica, and S.-H.H. Tye, J. High Energy Phys. 07, 051 (2002).

[17] S. Sarangi and S.-H.H. Tye, Phys. Lett. B 536, 185 (2002).

[18] N. Jones, H. Stoica, and S.-H.H. Tye, hep-th/0303269.

[19] W.J. Percival et al., Mon. Not. R. Astron. Soc. 327, 1297 (2001).

[20] J. Polchinski, String Theory (Cambridge University Press, Cambridge, England, 1998).

[21] G. Shiu, S.-H.H. Tye, and I. Wasserman Phys. Rev. D 67, 083517 (2003); J.M. Cline, H. Firouzjahi, and P. Martineau, J. High Energy Phys. 11, 041 (2002); G. Felder, J. GarciaBellido, P.B. Greene, L. Kofman, A. Linde, and I. Tkachev, Phys. Rev. Lett. 87, 011601 (2001); G. Felder, L. Kofman, and A. Linde, Phys. Rev. D 64, 123517 (2001).

[22] A. Sen, J. High Energy Phys. 08, 010 (1998); 09, 023 (1998); E. Witten, ibid. 12, 019 (1998); P. Horava, Adv. Theor. Math. Phys. 2, 1373 (1999).

[23] A. Melchiorri and C. Odman, Phys. Rev. D 67, 021501(R) (2003)

[24] J. Kovac et al., Nature (London) 420, 772 (2002).

[25] L. Pogosian and T. Vachaspati, Phys. Rev. D 60, 083504 (1999).
[26] A. Gangui, L. Pogosian, and S. Winitzki, Phys. Rev. D 64, 043001 (2001).

[27] C.J.A.P. Martins and E.P.S. Shellard, Phys. Rev. D 54, 2535 (1996); 65, 043514 (2002).

[28] C. Contaldi, M. Hindmarsh, and J. Magueijo, Phys. Rev. Lett. 82, 679 (1999).

[29] E.J. Copeland, J. Magueijo, and D.A. Steer, Phys. Rev. D 61, 063505 (2000).

[30] U.-L. Pen, U. Seljak, and N. Turok, Phys. Rev. Lett. 79, 1611 (1997); N. Turok, U.-L. Pen, and U. Seljak, Phys. Rev. D 58, 023506 (1998).

[31] L. Pogosian, Int. J. Mod. Phys. A 16S1C, 1043 (2001).

[32] U. Seljak, U.-L. Pen, and N. Turok, Phys. Rev. Lett. 79, 1615 (1997).

[33] U. Seljak and M. Zaldarriaga, Astrophys. J. 469, 437 (1996).

[34] M. Zaldarriaga and U. Seljak, Phys. Rev. D 58, 023003 (1998); L. Knox and Y.-S. Song, Phys. Rev. Lett. 89, 011303 (2002).

[35] I. Zehavi et al., astro-ph/0301280.

[36] J.-H.P. Wu, astro-ph/0012206; in Relativistic Astrophysics, edited by J. Craig Wheeler and Hugo Martel, AIP Conf. Proc. No. 586 (AIP, Melville, NY, 2001), p. 211.

[37] L.L. Cowie and E.M. Hu, Astrophys. J. Lett. 318, L33 (1987); E.M. Hu, ibid. 360, L1 (1990); M. Sazhin et al., astro-ph/0302547.

[38] T. Damour and A. Vilenkin, Phys. Rev. D 64, 064008 (2001).

[39] V.M. Kaspi, J.H. Taylor, and M.F. Ryba, Astrophys. J. 428, 713 (1994).

[40] A.N. Lommen and D.C. Backer, Astrophys. J. 562, 297 (2001); A.N. Lommen, astro-ph/0208572.

[41] A.H. Jaffe and D.C. Backer, Astrophys. J. 583, 616 (2003).

[42] The 2dFGRS website. 